\title{
Dictynna
}

Dictynna

Revue de poétique latine

11 | 2014

Varia

\section{La réception d'Empédocle dans la poésie latine : Virgile (Buc. 6), Lucrèce, Gallus et les poètes élégiaques}

Jacqueline Fabre-Serris

\section{(2) OpenEdition}

Journals

Édition électronique

URL : http://journals.openedition.org/dictynna/1045

DOI : $10.4000 /$ dictynna. 1045

ISSN : 1765-3142

Référence électronique

Jacqueline Fabre-Serris, «La réception d'Empédocle dans la poésie latine : Virgile (Buc. 6), Lucrèce, Gallus et les poètes élégiaques », Dictynna [En ligne], 11 | 2014, mis en ligne le 18 décembre 2014, consulté le 10 septembre 2020. URL : http://journals.openedition.org/dictynna/1045 ; DOI : https:// doi.org/10.4000/dictynna.1045

Ce document a été généré automatiquement le 10 septembre 2020.

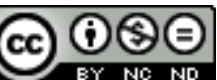

Les contenus des la revue Dictynna sont mis à disposition selon les termes de la Licence Creative Commons Attribution - Pas d'Utilisation Commerciale - Pas de Modification 4.0 International. 


\title{
La réception d'Empédocle dans la poésie latine : Virgile (Buc.6), Lucrèce, Gallus et les poètes élégiaques
}

\author{
Jacqueline Fabre-Serris
}

1 Cet article s'inscrit dans le cadre des travaux sur la réception des savoirs grecs à Rome, en l'occurrence, de la philosophie, et vise plus particulièrement à mettre en évidence, en prenant l'exemple des élégiaques, le rôle joué alors par les poètes dans les débats idéologiques de la fin de la République et du début du Principat. Les études sur la réception d'Empédocle ont porté principalement sur Ennius, Lucrèce et Virgile ${ }^{1}$. Je voudrais montrer, à partir d'une série d'analyses de textes de Virgile, de Lucrèce et des poètes élégiaques, que le genre littéraire inventé par Gallus témoigne lui aussi de l'intérêt pour Empédocle qui se manifeste à partir des Annales, et, bien plus, que la lecture faite par Gallus, non du philosophe directement mais de la réception de ce dernier chez Lucrèce, a joué un rôle fondamental dans la genèse des grands motifs élégiaques. Cette dimension philosophique dans un genre qui s'est présenté lui-même comme mineur atteste une des visées de la littérature de la fin du $1^{\text {er }}$ siècle av. J-C. : se constituer, par le biais de dialogues entre poètes, genres et textes, en espace de discussions et réflexions dans une société en pleine rénovation du fait de la crise politique et morale qui a résulté des conquêtes.

2 Je partirai de la Bucolique 6, qui contient, me semble-t-il, des indices laissant supposer qu'il y avait dans les Amores de Gallus des renvois à la philosophie d'Empédocle ou plus exactement à la lecture qu'en avait faite Lucrèce. L"hypothèse que je défendrai d'abord est que Virgile tient compte et réagit à 'cette réception élégiaque' d'Empédocle, qui se poursuivra et s'amplifiera chez les successeurs de Gallus. 


\section{La Bucolique 6 : les indices d'une présence d'Empédocle dans les Amores de Gallus}

3 Le premier passage de Virgile où l'on a identifié un renvoi à Empédocle se trouve dans le chant attribué à Silène dans la Bucolique 6. Le texte de cette églogue est, comme c'est souvent le cas dans les Bucoliques, tissé de jeux intertextuels compliqués dont le lecteur est implicitement invité à débrouiller les fils pour en saisir l'enjeu et les prises de position. Dès les premiers vers Virgile y affirme sa persona en tant que poète. Il se proclame le premier à avoir écrit à Rome de la poésie pastorale sur le modèle de Théocrite et donne toute solennité à cette déclaration en recourant à une mise en scène où il utilise deux moments du début des Aitia: l'attaque contre les Telchines ${ }^{2}$ et l'intervention d'Apollon Lycien auprès de Callimaque ${ }^{3}$, qu'il adapte à son cas personnel ${ }^{4}$ :

Cum canerem reges et proelia, Cynthius aurem uellit et admonuit : « Pastorem, Tityre, pinguis pascere oportet ouis, deductum dicere carmen. » (3-5)

«Alors que j'allais chanter les rois et les combats, le dieu du Cynthe me tira l'oreille et m'admonesta : « Un berger, Tityre, doit faire paitre de grasses brebis, mais son chant doit être finement tissé.»

Il associe le «tropos " prôné par Callimaque dans sa réponse aux Telchines, évoqué ici par l'expression carmen deductum, au genre pastoral, qu'il oppose à l'épopée (cum canerem reges et proelia). Ce passage programmatique est suivi d'un envoi à Varus, dont Virgile aurait pu chanter les exploits guerriers, puis d'un développement pastoral, implicitement présenté comme un exemplum du type de production poétique ordonné par Apollon. Le rapport à Varus est réduit à une mise en tête de son nom, proclamée agréable au dieu (11-2). Dans les vers 31-81, attribués à Silène, Virgile a inséré une variation sur le troisième passage fameux du début des Aitia: la rencontre en songe de Callimaque avec les Muses, conçue elle-même sur le modèle de celle d'Hésiode avec les mêmes déesses. L'objet en est la consécration poétique de Gallus, le fondateur d'un 'autre' nouveau genre, l'élégie. Conduit par une Muse sur les monts d'Aonie, Gallus est accueilli par le chœur de Phébus et Linus lui remet les chalumeaux par lesquels Hésiode " faisait descendre les ornes raides des montagnes" (rigidas deducere montibus ornos, 71), en lui enjoignant de dire « l'origine du bois de Grynium » :

His tibi Grynei nemoris dicatur origo ne quis sit lucus quo se plus iacet Apollo. (72-3)

"Qu'ils (les pipeaux d'Hésiode) te servent à dire l'origine du bois de Grynium afin qu'il n'y ait pas de bois sacré dont Apollon soit plus fier. »

Cette scène de consécration et d'inspiration poétique ${ }^{5}$ renvoie, si l'on en juge par sa variation chez Properce ${ }^{6}$, à un ou plusieurs précédents dans les Amores de Gallus, devenus célèbres à la fois pour leur imagerie mythologique (composée des Muses, de Phébus, de Linus, d'Hésiode et d'Orphée ${ }^{7}$ ) et pour les termes employés (una sororum, chorus par exemple) ${ }^{8}$. Je voudrais, avant de poursuivre, souligner l'importance de ce passage. L'ensemble de la Bucolique 6 est, en raison de cette scène, à interpréter dans une double perspective : de positionnement et de compétition vis-à-vis du seul poète cité nommément dans le texte, Caius Cornelius Gallus. Ce dernier avait, avec la parution de ses Amores, créé un événement en raison de la façon, nouvelle par rapport aux neoteroi, dont il exploitait la tradition poétique hellénistique et sa réception à Rome. 
C'est à lui que Virgile se mesure en quelque sorte quand il 'invente', à son tour, un genre littéraire, inspiré de la Grèce.

Silène, qui est sans doute un personnage tiré des Amores $^{9}$, est assimilé ici à Orphée ${ }^{10}$ par l'évocation de l'effet qu'il produit sur les faunes, bêtes sauvages et chênes (27-8) ${ }^{11}$, ce qui en fait implicitement l'alter ego de Gallus. C'est au début de son chant que Virgile renvoie de façon seconde à Empédocle par le biais d'un renvoi premier au chant cosmogonique qu'Apollonios de Rhodes attribue à Orphée au livre 1 de ses Argonautiques. Son Namque canebat uti ("et en effet il chantait comment ... », 32) a été reconnu comme une transcription latine du début du vers 496 du livre 1 des

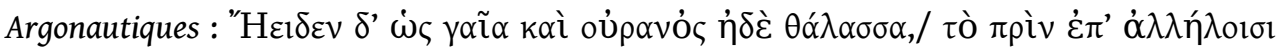

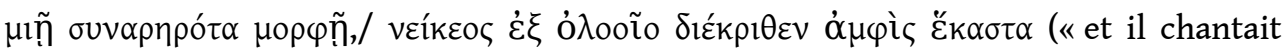
comment la terre et le ciel et la mer qui à un moment étaient rassemblés en une forme unique, furent séparés les uns des autres par la discorde... », 496-8). Dans ce passage, la naissance de l'univers est présentée comme résultant d'une séparation des éléments sous l'effet de la Discorde, le principe qui, dans le système cosmique empédocléen, est opposé à l'Amour ${ }^{12}$. Le namque canebat uti amorce aussi une cosmogonie où Virgile combine un renvoi à Lucrèce (un autre poète ayant utilisé Empédocle) par le biais de la mention du vide (per inane, 31) et le recours aux termes uti, semina, exordia ${ }^{13}(31,32,33)$, avec deux allusions directes au philosophe sicilien lui-même : la mention des quatre éléments (terrarum, animae, maris et ignis, 32-3) et l'usage d'une technique typique du style d'Empédocle: l'évocation de ces quatre éléments sous la forme de leurs manifestations dans la nature : la terre avec le mot solum (35), la mer avec celui de ponto (35), le feu sous l'aspect du soleil (37) et l'air avec les nuages (38) ${ }^{14}$. Comme chez Apollonios, le passage se termine par la mention des êtres vivants (animalia, 40); chez

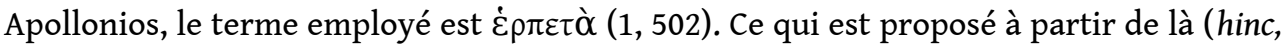
41), c'est une suite au chant de l'Orphée d'Apollonios de Rhodes, selon un schéma qui a été reconnu comme renvoyant à Hésiode. À cette cosmogonie succèdent en effet des histoires mythologiques, mais dont les premières seulement sont reprises de l'auteur de la Théogonie (41-2) ${ }^{15}$, ce qui suggère l'existence d'un autre modèle, qu'il est tentant d'identifier avec le poète honoré ensuite à la fois comme le successeur romain d'Hésiode et l'équivalent d'Orphée.

7 S'il prend en compte les jeux intertextuels de la Bucolique 6, le lecteur moderne (qui n'a pas accès aux Amores) est, me semble-t-il, amené à se poser une question : les renvois à Empédocle doivent-ils, eux aussi, être mis en relation avec Gallus du moment que c'est le cas pour les allusions à Hésiode, Orphée, Callimaque, Lucrèce et (sans doute) Apollonios ? Il est probable que l'auteur de la Théogonie et Orphée aient été évoqués par Gallus dans une ou des scènes d'inspiration poétique. C'est ce que laissent supposer, quand on les confronte, la consécration poétique de Gallus dans la Bucolique 6 et la valorisation que fait Properce de sa propre modulation du genre élégiaque dans l'élégie 2,13, qui combine des allusions à Hésiode, à Orphée et à Linus, avec un renvoi à des vers de Gallus découverts dans le papyrus de Qașr Ibrîm ${ }^{16}$. Callimaque est un des modèles revendiqués par Gallus : pour prendre un exemple, un de ses plus fameux exempla mythologiques, l'histoire d'Acontius, était repris des Aitia ${ }^{17}$. C'est apparemment à Lucrèce que l'auteur des Amores a emprunté des motifs essentiels de sa poétique comme le furor, l'absence de remèdes à l'amour ou des expressions comme digna carmina $^{18}$. Il est aussi vraisemblable que l'héroïne des Argonautiques, Médée ${ }^{19}$ ait fait partie des exempla galliens ${ }^{20}$. Si on peut ajouter Empédocle à cette série, le choix des 
allusions insérées par Virgile dans la Bucolique 6 se justifierait alors par la façon dont fonctionne cette technique chez les poètes latins, qui renvoient à la fois à un auteur, en l'occurrence au poète loué au centre du texte: Gallus, et aux auteurs auxquels renvoyait ce dernier, avec une perspective qui peut être soit de conforter son point de vue en adoptant la même perspective, soit de polémiquer avec lui, ce qui est à mon avis le cas ici.

8 La thèse que je vais défendre est en effet que les renvois à Empédocle dans la Bucolique 6 sont à replacer dans le contexte polémique d'une poésie savante, où tout fait sens, et qu'en l'occurrence, la façon dont Gallus a utilisé le philosophe d'Agrigente fait partie des éléments constitutifs de la prise de position critique de Virgile vis-à-vis des Amores. Les renvois à Empédocle (via Apollonios de Rhodes) étant ici associés à des renvois à Lucrèce, mon hypothèse de départ a été que l'usage d'Empédocle chez Gallus, qui a repris, au Lucrèce analyste de l'amour, divers motifs et expressions en les orientant différemment puisque sa perspective sur la passion est antagoniste de celle du philosophe épicurien, est passé par la réception d'Empédocle dans le De rerum natura ${ }^{21}$. Je développerai ce point de vue en trois points. J'analyserai d'abord le conseil adressé par Virgile à Gallus tel qu'il découle de sa mise en pratique dans le chant de Silène, où est proposée une lecture d'Empédocle médiatisée par Apollonios, dont la vision négative de l'évolution de l'amour trouvait un écho dans les conceptions développées par Lucrèce sur la passion, auxquelles adhère pour sa part Virgile. Puis, après avoir retracé dans ses grandes lignes 'la réception lucrétienne' d'Empédocle susceptible d'avoir influencé Gallus, j'essayerai de reconstituer la façon dont ce dernier l'a reprise dans les Amores, en la modifiant dans sa propre perspective. Je me servirai pour cela de textes élégiaques renvoyant nominativement ou indirectement à lui. Ma dernière partie sera consacrée à l'analyse de la façon dont les poètes élégiaques ont exploité les motifs galliens, prenant ainsi position dans le débat instauré par Virgile avec l'auteur des Amores, et à la façon dont ils lui ont répondu, ainsi qu'à Lucrèce.

\section{Conseil d'ami : qu'est-ce qu'on peut inférer de la lecture d'Empédocle proposée par Virgile à Gallus ?}

Comment le chant de Silène est-il à la fois une illustration du choix générique fait par Virgile à la suite de l'intervention d'Apollon, en même temps qu'une alternative et une réponse au choix générique effectué préalablement par Gallus? Pour répondre à cette question, il faut préalablement analyser l'excursus consacré à l'auteur des Amores. C'est lorsqu'il est en train d'errer au bord du Permesse (Virgile a choisi un comportement typique de l'amant en proie aux souffrances amoureuses et un lieu symbolique de l'inspiration élégiaque ${ }^{22}$ ) qu'une Muse vient chercher Gallus. Elle l'amène dans un autre lieu, les monts d'Aonie, associés à l'inspiration hésiodique, où Linus l'enjoint de traiter un sujet étiologique à la manière du vieillard d'Ascra : l'origine du bois de Grynium. Comme ces mots sont prononcés au cours d'une scène de consécration poétique, conçue sur le modèle de celle de la Théogonie, où Hésiode reçoit des Muses le sujet de son poème, même s'ils constituent une suggestion de Virgile, incitant Gallus à changer d'inspiration, cette suggestion a nécessairement un fondement dans l'œuvre du poète dont la valeur est alors reconnue par Apollon et son chœur. Deux autres textes permettent, me semble-t-il, de préciser la nature du texte (ou du passage) gallien auquel Virgile renvoie : le livre 10 des Métamorphoses d'Ovide et le Culex ${ }^{23}$, auxquels on 
peut ajouter certaines allusions dans les Bucoliques 3, 5 et $10^{24}$. Les Métamorphoses et le Culex offrent une description de bois conçue sur le même modèle : une énumération d'espèces d'arbres, accompagnée, parfois, de quelques vers faisant allusion à l'origine' de certains d'entre eux, origine chaque fois expliquée par le destin malheureux de tel ou tel mortel, en proie à des souffrances, liées à l'amour au sens large. Une partie de ces arbres est commune au texte d'Ovide et au Culex: le peuplier, le chêne, le cyprès, le hêtre et le myrte, ce qui laisse supposer une même source. Dans les Métamorphoses, ce bois est évoqué dans un contexte gallien : il constitue le cadre d'un chant d'Orphée, construit comme une réponse à Virgile et à sa critique de la poétique gallienne au livre 4 des Géorgiques ${ }^{25}$. Le Culex est un texte datant probablement du $1^{\text {ier }}$ siècle ap. J.-C. dont l'auteur fait semblant d'être de l'époque d'Octave, et où la critique a identifié un certain nombre de renvois à Gallus ${ }^{26}$. Il est assez probable que les éléments communs à son auteur et à Ovide aient été tirés d'un passage ou d'un poème de Gallus consacré au bois de Grynium ${ }^{27}$.

10 Après son début cosmogonique, placé sous le signe d'Empédocle et de Lucrèce, le chant de Silène se poursuit par une série d'évocations rapides d'histoires mythologiques, soit érotiques, soit se terminant par une métamorphose. Ce dernier élément le rapproche du poème gallien sur l'origine du bois de Grynium. Dans la mesure où le chant de Silène est l'exemple du type de carmen deductum conseillé par Apollon à Virgile, on peut supposer que ce dernier l'a conçu de façon à montrer au poète des Amores une façon de traiter des sujets cosmogoniques, érotiques et étiologiques différente de celle que ce dernier avait choisie. Que le chant de Silène soit une réponse à la poétique gallienne et, entre autres poèmes, à celui sur le bois de Grynium est, me semble-t-il, 'signalé' par la présence de deux histoires traitées par Gallus : celle d'Atalante, un des exempla les plus fameux des Amores (mais Virgile choisit la version qui associe l'héroïne à Hippomène et non à Milanion comme c'était le cas chez Gallus) et celle des Héliades, vraisemblablement présentes dans le texte sur le bois de Grynium ${ }^{28}$. Ce n'est pas un hasard si Atalante et les Héliades sont évoquées successivement, juste avant la scène de consécration poétique de Gallus.

11 Les récits qui composent la deuxième partie du chant de Silène ont un point commun, qui semble avoir été aussi celui des destins des mortels évoqués dans le poème sur l'origine du bois de Grynium, et qui, de façon plus générale, correspond à la poétique élégiaque centrée sur le furor et ses effets. Tous évoquent des mortels victimes d'un 'excès' de passion: désir érotique dans le cas d'Hylas, enlevé par les nymphes d'une source, et appelé vainement par ses compagnons; folie amoureuse pour Pasiphaé, éprise d'un taureau; attirance pour des pommes d'or dans le cas d'Atalante, qui du coup fut vaincue à la course ; douleurs du deuil pour les sœurs de Phaéthon ; vengeance pour Scylla, métamorphosée en monstre qui détruisit les bateaux d'Ulysse ; vengeance également pour Philomèle, changée en oiseau après le repas qu'elle offrit à son mari ${ }^{29}$. C'est l'épisode le plus développé, l'amour de Pasiphaé pour un taureau, qui donne le sens de l'ensemble. Virgile y intervient en s'adressant à la jeune femme, qu'il plaint tout en condamnant sa passion: A! uirgo infelix, quae te dementia cepit! («Ah! malheureuse vierge, quelle démence t'a saisie !», 47). Pasiphaé est déclarée par trois fois infortunée: et fortunatam si numquam ... (45), a! uirgo infelix $((47,52)$, une exclamation dans laquelle on a reconnu un renvoi à l'Io de Calvus. Sa vie se réduit à une errance à la recherche de l'être aimé (tu nunc in montibus erras, 52 ; errabunda bouis uestigia, 58), ce qui est exactement le cas de Gallus sur les bords du Permesse ${ }^{30}$. Si Virgile 
se situe ici globalement dans la ligne de Lucrèce en condamnant la passion en soi pour ces retombées négatives dans la vie des hommes, les dernières histoires (celles de Scylla et de Philomèle) où l'amour se transforme en haine et colère, sont sans doute à lire à la lumière de la réception d'Empédocle dans les Argonautiques, où les deux principes empédocléens, qui expliquaient dans le chant d'Orphée la naissance du monde, trouvent une illustration morale dans l'histoire mythologique racontée : la passion de Médée pour Jason, évoluant précisément en colère et haine ${ }^{31}$.

Outre que cette vision des effets de l'amour correspond au point de vue de Virgile sur les méfaits de la passion, il y avait, je crois, un autre intérêt à renvoyer à Apollonios de Rhodes en tant que lecteur d'Empédocle. C'était aussi une alternative au choix de l' 'autre' poète grec 'empédocléen' : Homère, dont le passage sur les amours de Mars et de Vénus avait été lu comme une illustration des principes cosmogoniques du philosophe sicilien. Or les amours de Mars et de Vénus sont l'épisode mythologique évoqué par Lucrèce dans le prologue de son texte, qui, selon D. Sedley, a été mis sous le signe d'Empédocle. La thèse que ce dernier a soutenu, d'une façon tout à fait convaincante, est, je le rappelle, que «the proem of the $D R N$, is, and is meant to be recognized, as an imitation of the proem of Empedocles' physical poem ${ }^{32} »$. Pourquoi une alternative ? parce que - c'est la thèse que je vais maintenant défendre - l'épisode homérique avait été repris également par Gallus, qui utilisait 'à sa façon' la lecture empédocléenne qu'en avait faite Lucrèce. Après une analyse rapide de deux textes laissant supposer que Gallus évoquait les amours de Mars et de Vénus, je rappellerai la lecture qu'en a proposée Lucrèce en essayant de montrer pourquoi elle a intéressé Gallus, puis je proposerai une hypothèse sur ce que l'on peut inférer, à partir de plusieurs poèmes élégiaques, sur le traitement de cette histoire par le fondateur de l'élégie.

\section{Indices en faveur d'un traitement gallien des amours de Mars et de Vénus}

13 Le premier passage que je prendrai se trouve au chant 4 des Géorgiques. Les amours de Mars et de Vénus sont brièvement évoquées dans les vers qui racontent la venue d'Aristée chez sa mère Clyméné. Ces vers précèdent l'histoire d'Orphée, qui est traitée comme un exemplum élégiaque ${ }^{33}$. Dans sa description de la visite d'Aristée à sa mère, Virgile a inséré divers renvois aux Amores. Le premier est constitué par les noms de trois nymphes de l'entourage de Clyméné. Il est question, au vers 339, de Cydippé (c'est l'aimée d'Acontius), au vers 339 aussi, de Lycorias (son nom rappelle celui de Lycoris, la puella de Gallus) et au vers 344 d'Aréthuse (cette nymphe arcadienne est évoquée au début de la Bucolique 10, où l'adresse à Gallus implique des reprises textuelles des Amores). Le deuxième renvoi se trouve au vers 369, où Virgile mentionne l'Hypanis parmi les fleuves contemplés alors par Aristée : l'Hypanis (saxosusque sonans Hypanis... : "l'Hyspanis sonore à cause de ses rochers"; 4, 369). Le seul vers de Gallus, connu avant la découverte du papyrus de Qasr Ibrîm: uno tellures diuidit amne duas (" il partage deux terres au moyen d'un seul fleuve »; fr. 1 Morel et Courtney) concernait précisément ce fleuve, séparant l'Asie et l'Europe.

14 C'est à propos de l'activité attribuée à Clyméné (assise au milieu des nymphes qui filent, elle leur raconte des histoires) que Virgile fait allusion aux amours de Mars et de Vénus : Inter quas curam Clymene narrabat inanem/Volcani Martisque dolos et dulcia furta/ aque 
Chao densos diuom numerabat amores ("parmi elles Clyméné racontait l'objet du vain souci de Vulcain, les ruses de Mars, et les doux larcins/sa liaison secrète et elle énumérait depuis le chaos les amours abondantes des dieux ", 4, 345-7). Dans les deux premiers vers, on peut relever trois termes élégiaques : curam $^{34}$, dolos, et dulcia furta. Ces choix linguistiques laissent supposer que l'emploi d'amores au vers suivant est à prendre comme un renvoi au titre du recueil de Gallus. Aussi est-il peu probable que la seule histoire mythologique évoquée par Virgile (en termes élégiaques donc) ne l'ait pas été préalablement par le fondateur de l'élégie. De fait le chant de Clyméné peut être considéré comme une variation sur celui de Silène, qui concentrerait ses deux parties en une seule. On a en position de fabula développée comme l'était l'aventure de Pasiphaé, et donnant, comme elle, le sens de la série mythologique, les amours de Mars et de Vénus, qui sont suivies d'autres histoires sur le même sujet ${ }^{35}$. Mais en raison à la fois de sa place au début du chant de Clyméné et de la lecture philosophique qu'on a faite de l'épisode homérique (la victoire de l'Amour sur la Discorde), cette fabula équivaut aussi à la partie cosmogonique du chant de Silène. La conception philosophique évoquée : celle d'Empédocle est la même que dans la Bucolique 6, mais la visée de Clyméné est différente. Des quelques mots qu'en dit Virgile, il s'agirait d'une valorisation de la victoire de l'amour, non d'une condamnation de la passion, ce qui pourrait être donc, aussi, le point de vue choisi par Gallus. La suite confirme qu'on a dans ce passage des Géorgiques une variation sur la sixième églogue. On y trouve une situation répétant celle qui précède le chant de Silène. Protée est présenté comme un berger ( 395 ; 433-6) ; comme le compagnon de Dionysos, il faut qu'il soit surpris dans son sommeil et garrotté pour accepter de délivrer son chant ${ }^{36}$.

L'élégie 2, 32 de Properce confirme, me semble-t-il, que Gallus utilisait comme exemplum l'histoire des amours de Mars et de Vénus. Properce y défend la réputation de Cynthie en arguant de trois exempla: celui d'Hélène qui ne pâtit pas d'avoir quitté sa patrie par amour pour un étranger (31-2), celui de Vénus, qui, quoiqu'elle ait été séduite par Mars, n'en fut pas moins honorée dans le ciel (33-4), et celui d'ÆEnone, une nymphe, qui devint l'amante du berger Pâris, sans être blâmée par ses sœurs Hamadryades, les Satyres et Silène (35-40). Il y a des chances pour que le premier exemple ait été présent dans les Amores: Pâris et Hélène sont les protagonistes d'une des trois Héroïdes doubles d'Ovide, dont les deux autres ont des épistoliers reconnus comme galliens : Héro et Léandre ; Acontius et Cydippé. Il est aussi probable que Gallus ait utilisé comme exemplum les amours de Pâris et d'Enone. Elles font partie des histoires racontées par Parthénius dans le recueil qu'il dédia à Gallus pour qu'il s'en serve dans ses poèmes ${ }^{37}$. C'est également le sujet de l'Héroïde 5 d'Ovide, qui le traite à la manière gallienne ${ }^{38}$. L'épisode central: les amours de Mars et de Vénus sert apparemment à justifier, en tant qu'exemplum chez les dieux, des comportements identiques chez les hommes ${ }^{39}$. L'impression est à une variation sur l'épisode homérique, où Vulcain visait, sans succès, à discréditer les deux amants, Mars et Vénus étant ici implicitement utilisés comme garants pour les liaisons humaines illicites, autrement dit, pour l'amour élégiaque. Le traitement ovidien des amours de Mars et de Vénus dans l'Art d'aimer va dans le même sens que l'élégie de Properce : quand Vulcain qui les a pris au piège convoque tous les dieux pour qu'ils voient le spectacle (Mars et Vénus sont enveloppés nus dans un filet), loin de blâmer les deux amants, l'un d'entre eux propose en riant à Mars de prendre sa place si ses chaînes lui pèsent (2, 585-6). J'en reviens maintenant au De rerum natura, dont le début est placé sous le signe d'Empédocle, si on souscrit aux analyses de D. Furley et de D. Sedley ${ }^{40}$. 


\section{La lecture lucrétienne des amours de Mars et de Vénus}

D. Sedley considère que les renvois de Lucrèce à Empédocle sont seulement formels. L'objectif du philosophe épicurien serait de se présenter « as the Roman Empedocles the great Roman poet of nature ${ }^{41} »$ d'un point de vue uniquement littéraire ${ }^{42}$. En tant que poéticienne, je trouve peu crédible l'idée qu'il y aurait, chez un poète-philosophe, dans un passage aussi programmatique qu'un prooemium, des éléments renvoyant à un autre poète-philosophe d'une façon seulement formelle. Dans la poésie latine la technique des renvois implique un jeu avec le texte source : les mots et/ou motifs repris sont insérés dans un contexte nouveau, ce qui implique des variations et des adaptations, voire une prise de position polémique ${ }^{43}$. Si on admet l'hypothèse d'un début calqué sur celui d'Empédocle (un hymne à Aphrodite qui aurait ouvert son poème) ou des échos à des passages du Peri Phuseos, la difficulté est, comme toujours, dans la pratique intertextuelle romaine, de faire le partage entre reprise et innovation ${ }^{44}$, et elle est accrue ici par le fait que nous n'avons pas le prologue imité. S. Trépanier observe qu'il n'y avait pas de précédent, ni empédocléen ni épicurien, pour la scène d'amour entre Mars et Vénus décrite par Lucrèce. Pour deux raisons en ce qui concerne Empédocle : l'Amour est invisible (B. 17.26) et il ne s'unit jamais à la Discorde, mais alterne avec elle ou lui laisse la place (B. $35.12-3)^{45}$. À son avis, l'adaptation lucrétienne a consisté à utiliser la scène homérique des amours de Mars et de Vénus. Il s'agit, comme je l'ai rappelé, d'un épisode dont les Anciens avaient fait une lecture empédocléenne en y voyant une figuration de la victoire de l'Amour. Il me semble important de souligner que Lucrèce a choisi de renvoyer par là à un exemple de la 'réception' d'Empédocle, et non au texte même du philosophe. D'où le fait qu'il n'y a pas de correspondance exacte sur le plan de la théorie : si Vénus et Mars figurent les deux forces cosmiques empédocléennes, l'Amour et la Discorde, l'évocation de leurs rapports, aussi bien chez Homère que chez Lucrèce, ne correspond pas à la cosmologie du philosophe sicilien, chez qui l'Amour et la Discorde s'affrontent et alternent. C'est peut-être une des raisons pour lesquelles Lucrèce a choisi de reprendre l'épisode homérique : il lui permettait de se livrer à des variations personnelles ${ }^{46}$.

De fait, la version que Lucrèce en donne correspond à une vision proprement romaine de la déesse. Dans un article consacré aux étymologies que l'on trouve chez Varron, S. Hinds a fait observer que «the name of Venus seems variously to be linked in ancient etymologizing contexts with uincire, uincere, uis, uenire and uendere, as well as with its true cognates uenia, uenerari and uenenum ${ }^{47}$ ». Dans le prooemium du De Rerum natura, il relève deux étymologies uenire et $u i^{48}$. Lucrèce, me semble-t-il, en a utilisé aussi deux autres : uincere et uincire. Pour résumer le résultat en une phrase : il est question, dans son prooemium, de la 'force'(ui, 14) de Vénus, qui quand elle 'vient'(uenio : aduentum, 7), met en mouvement tous les êtres de l'univers, et 'vainc' (uincere: deuictus, 34) Mars, qu'elle 'lie' (uincire) dans ses bras. Certes on ne trouve pas uincire dans le prooemium, mais il y a, à la place, l"image' longuement décrite de la déesse enlaçant le dieu (... tuo recubantem corpore sancto/ circumfusa super... 37-8 ${ }^{49}$ ) qui vaut, je crois, pour équivalent du mot. On a là, par ailleurs, une préfiguration des analyses que Lucrèce développera ensuite sur la passion amoureuse, mais dans une perspective négative, conforme à la conception épicurienne. 

pour nous » (haec Venus est nobis, 1058), d'où il ressort qu'une fois que Vénus (le désir) a 'vaincu', quand l'amant devient incapable de se tourner vers d'autres objets, la visée de ce dernier est d'être 'lié' à l'autre/la femme en l'occurrence, au plus près. On trouve les termes uis (Veneris ... uis, 1172) et uincere (uincat, 1119) et surtout un grand nombre de variations sur l'idée de liens : usque adeo cupide in Veneris compagibus haerent (" tant sous l'effet du désir ils restent attachés dans les liens de Vénus », 1113); captum retibus ipsis ... et ualidos Veneris perrumpere nodos (" pris dans ces filets eux-mêmes ... et de rompre les nœuds solides de Venus ", 1147-8); complexa uiri corpus cum corpore iungit (« enlaçant de son corps le corps de l'homme elle l'unit au sien», 1193); Nonne uides etiam quos mutua saepe uoluptas/ uinxit, ut in uinclis communibus excrucientur? (" Ne voistu pas à quel point même ceux qu'une volupté mutuelle a souvent enchainés sont dans leurs chaînes communes mis à la torture ? ", 1201-2) ; ualidis Veneris compagibus haerent («ils restent attachés aux assemblages solides que Vénus a créés », 1205), uinctos tenere (« les tenir enchaînés », 1206) ${ }^{50}$. Un détail qui a son importance : le désir de lier avec son corps le corps de son partenaire, attribué à la femme (complexa uiri corpus cum corpore iungi), n'est pas sans évoquer l'image' du corps de Vénus enveloppant celui de Mars au livre 1. La différence est que la scène décrite au livre 1 a une connotation positive, essentiellement liée à la lecture historique qu'en a faite Lucrèce, peut-être en relation avec la façon dont (si l'on considère que le De rerum natura est une épopée d'un nouveau genre) son autre grand modèle littéraire: Ennius a pu utiliser la doctrine empédocléenne ${ }^{51}$. Si l'on se réfère au fragment 225-6 (Skutsch) des Annales (postquam Discordia taetra/ belli ferratos postes portasque refregit : « après que l'horrible Discorde eut brisé les jambages bardés de fer des portes de la guerre ») il semble que Ennius ait expliqué par la domination de la Discorde un moment fondamental de l'histoire romaine ${ }^{52}$.

Amores de Gallus exploraient, comme leur titre l'indique, ce qu'est l'amour et sa place dans la vie humaine, à travers une série de motifs, dont deux parmi les plus importants étaient présents dans le De rerum natura : la notion de furor $(4,1069,1117)$ et l'idée qu'il n'est pas de remèdes à l'amour (1115-20). L'hypothèse que je voudrais développer maintenant est qu'on peut ajouter à ces motifs le déploiement thématique des deux étymologies de Vénus associées à la scène perçue comme empédocléenne du prooemium du De rerum natura: uincere et uincire. La première étymologie (uincere) a donné lieu à la thématique de la victoire de l'Amour et des combats de Vénus opposés aux guerres menées par les Romains, évoquées dans le papyrus de Qaṣr Ibrîm par les trophées accrochés aux temples. La seconde (uincire) à la thématique de la première nuit d'amour, celle qui 'lie' les amants et initie le début de foedera (un terme lucrétien, emprunté peut-être à Empédocle ${ }^{53}$ ) souhaités éternels. Comme les rapports entre Amour et Discorde, ces foedera sont, dans l'élégie, soumis à d'incessantes fluctuations, une idée absente de la peinture lucrétienne de l'amour. 


\section{Traces de la réception gallienne d'Empédocle chez les élégiaques}

\section{L'amicus Sulpiciae et Sulpicia}

20 Je prendrai l'élégie 3, 8 du Corpus Tibullianum comme premier indice du fait que Gallus avait perçu les connotations empédocléennes de la mise en scène lucrétienne des amours de Mars et de Vénus, et repris cet exemplum mais en l'orientant dans une perspective différente de celle du philosophe épicurien ${ }^{54}$. La critique attribue ce texte à un auteur inconnu, généralement désigné sous le terme d'amicus Sulpiciae. La fiction imaginée par ce poète pour faire l'éloge de Sulpicia est une variation sur le début du De rerum natura. Il imagine qu'à l'occasion des Matronalia, la fête célébrée en son honneur, Mars viendra dans son temple pour voir Sulpicia mais qu'il risque d'être vaincu par l'amour, Sulpicia prenant pour l'occasion la place de Vénus sans que celle-ci ne s'en offusque. On retrouve, déplacés sur Mars et sur Sulpicia, des termes ou des motifs évoquant certaines des étymologies associées par Lucrèce au nom de Vénus au début du De rerum natura: uenio (spectatum e caelo, si sapis, ipse ueni : "pour la voir, viens en personne du ciel, si tu es sage », 2 ; seu ... uenit, soit qu'elle vienne »,12) ; uis (le dieu est qualifié de uiolente au vers 3 ) ; uincere (à la vue de Sulpicia Mars risque de laisser tomber ces armes sous l'effet de l'admiration :... caueto/ ne tibi miranti turpiter arma cadant, 3-4), auxquelles l'auteur a ajouté d'autres étymologies varronniennes : uenia (suggérée par l'expression hoc Venus ignoscet, "Vénus te le pardonnera », 3) et ueneror (comptis est ueneranda comis, « sa coiffure la rend digne d'être vénérée », 10).

21 Ces jeux étymologiques ont lieu sur un fond gallien. L'amicus Sulpiciae use de plusieurs mots du glossaire élégiaque constitué par le fondateur du genre : dignus et choro dans le même vers (dignior est uestro nulla puella choro, « aucune jeune fille n'est plus digne de votre chœur ", 24), uro (répété : urit... urit , 11-2) et Eois (20), associé au nom de Gallus et/ou de Lycoris dans les Amours (Gallus et Hesperiis et Gallus notus Eois/ et sua cum Gallo nota Lycoris erit, 1, 15, 29-30) et dans l'Art d'aimer (Vesper et Eoae nouere Lycorida terrae, 3, 537). Ces deux derniers mots sont par ailleurs utilisés aussi par Properce dans l'élégie 2, 3, 43-4 (uret et Eoos, uret et Hesperios), ce qui est le signe d'une source commune.

Pourquoi l'amicus Sulpiciae a-t-il repris le mythe des amours de Mars et de Vénus en jouant de toutes les étymologies latines de la déesse et en adaptant l'histoire au cas de Sulpicia? Sa variation reflète en fait celle qu'avait préalablement opérée Sulpicia ellemême dans sa première épigramme. Dans ce texte où elle se met dans la position de l'amant-poète célébrant sa puella, Sulpicia évoque en effet la 'venue' de l'amour (tandem uenit amor, 13,1) sous l'aspect de celle de Cérinthus, déposé dans ses bras par Vénus (illum Cytherea .../ attulit in nostrum deposuitque sinum, 3-4), autrement dit, exactement dans la position du Mars de Lucrèce. Si la variation sur les amours de Mars et de Vénus proposée dans l'élégie 3, 8 peut s'expliquer par le préalable de la première épigramme de Sulpicia (dont l'élégie 3, 8 constitue le pendant en tête du recueil), qu'en est-il de celle imaginée par Sulpicia sur ces mêmes amours? Je propose de la prendre comme un indice à la fois de leur rôle de 'mythe fondateur' dans l'élégie gallienne, et de la lecture 'empédocléenne' via Lucrèce qu'en faisait l'auteur des Amores.

Le deuxième texte que j'évoquerai à l'appui de mon hypothèse est l'élégie, 3, 11 du Corpus Tibullanium, due sans doute à Sulpicia elle-même ${ }^{55}$. Les renvois à Gallus se marquent d'abord dans le vocabulaire des vers 5-8 : 
Te nascente nouum Parcae cecinere puellis

seruitium et dederunt regna superba tibi.

Vror ego ante alias: iuuat hoc, Cerinthe, quod uror,

si tibi de nobis mutuus ignis adest ;

mutuus adsit amor, per te dulcissima furta

perque tuos oculos per Geniumque rogo.

«À ta naissance les Parques ont annoncé un esclavage sans précédent pour les jeunes filles et elles t'ont fait le don d'une domination orgueilleuse. Je brûle, moi, plus que toute autre; il me plaît, Cérinthus, de brûler s'il y a en toi à notre propos un feu mutuel ; que l'amour soit mutuel entre nous, je t'en conjure, au nom de nos très doux larcins, par tes yeux et par ton Génie. » amor et à une de ses métaphores, ignis, et l'expression dulcissima furta (présente aussi dans le passage des Géorgiques, 4 évoquant les amours de Mars et de Vénus, sous la forme dulcia furta). L'expression mutuus amor est développée ensuite dans quatre vers qui jouent sur l'étymologie uincire de Vénus. Ils développent le motif des 'liens' réciproques créés par le sentiment qu'incarne la déesse :

Nec tu sis inusta, Venus : uel seruiat aeque uinctus uterque tibi uel mea uincla leua sed potius ualida teneamur uterque catena nulla queat posthac quam soluisse dies (13-6)

«Toi, Vénus, ne sois pas injuste: fais que nous soyons esclaves, liés également par toi l'un et l'autre, ou bien allège mes liens ou plutôt fais que nous soyons tenus, l'un et l'autre, par une chaîne solide qu'aucun jour ensuite ne puisse délier. »

La mention du jour qui pourrait délier les uincla de l'amour laisse supposer un cadre nocturne. J'interprète ce passage comme indicatif du fait que Gallus associait à l'exploitation de l'étymologie uincire le motif de la nuit d'amour. Avant d'essayer de corroborer cette hypothèse en me servant d'autres textes élégiaques, je soulignerai qu'aux vers 3-4: te nascente nouum Parcae cecinere puellis/ seruitium et dederunt regna superba tibi, Sulpicia use, implicitement, d'une autre étymologie de Vénus : uincere, là aussi sous l'aspect d'un motif que Gallus lui associait: le seruitium amoris. L'idée de victoire est suggérée par le renvoi que constitue l'expression Parcae cecinere à l'élégie 1, 7 de Tibulle, où les Parques chantent le jour du triomphe de Messala sur les peuples d'Aquitaine (Hunc cecinere diem Parcae fatalia nentes/ stamina..., «c'est ce jour que les Parques ont chanté, en tissant leurs fils fatals », 1-2).

\section{Properce}

Tout texte adressé à un auteur contenant nécessairement des renvois à son œuvre, les élégies 1,10 et 1, 13 de Properce, qui sont non seulement adressées à Gallus, mais le mettent en scène, constituent aussi un témoignage intéressant. Or elles confirment la présence dans les Amores, du motif de la nuit d'amour (avec développement sur les liens de Vénus), l'élégie 1, 10 comportant en outre une prise de position contre Lucrèce.

L'élégie 1, 10 s'ouvre sur une scène dont Properce prétend avoir été le témoin, mais où meminisse (comme c'est généralement le cas) ${ }^{56}$ et peut-être aussi uidimus signalent des renvois textuels :

$O$ iucunda quies, primo cum testis amori affueram uestris conscius in lacrimis !

O noctem meminuisse mihi iucunda uoluptas o quotiens uotis illa uocanda meis, 
cum te complexa morientem, Galle, puella

uidimus et longa ducere uerba mora! (1-6)

« $O$ repos délicieux, quand, témoin de la première fois où vous avez fait l'amour, j'étais là et j'avais connaissance de vos larmes ! O volupté délicieuse de me souvenir de cette nuit (ô de combien de vœux j'ai dû l'appeler) quand je t'ai vu, Gallus, mourir au moment où ton amie t'enlaçait et finir par parler après un long moment. »

Le vers incluant meminisse renferme une expression iucunda uoluptas, utilisée par Lucrèce. C'est donc probablement au De rerum natura que meminisse renvoie. Dans le prooemium du livre 2, Lucrèce indique qu'il n'y a pas de iucunda uoluptas à voir quelqu'un être tourmenté (uexari) mais qu'il est doux (suaue) de regarder les maux auxquels on échappe soi-même (2,1-4). L'élément à retenir ici (et qui est une des raisons du renvoi de Properce) est la situation de spectateur des passions d'autrui, dont l'image de la tempête est une métaphore, comme le confirment les vers 9-10, où il est question des sages abaissant leurs regards sur les hommes en train d'errer à la recherche du chemin de la vie. Cette situation de spectateur détaché est aussi celle de Lucrèce au livre 4 dans les divers passages où il décrit l'acte amoureux en usant à plusieurs reprises du mot uoluptas $(1075,1081,1085,1114)$ chaque fois dans des contextes d'où il ressort qu'elle n'est pas iucunda, et en particulier dans ce passage (que j'ai déjà cité) à propos des liens du désir : Nonne uides etiam quos mutua saepe uoluptas/ uinxit, ut in uinclis communibus excrucientur (" ne vois-tu pas à quel point, même ceux qu'une volupté mutuelle a souvent enchaînés sont dans leurs chaînes communes mis à la torture? ", 1201-2 »).

Que Properce réponde ici à Lucrèce en célébrant une première nuit d'amour de Gallus, qualifiée de iucunda quies, et en revendiquant pour son propre compte une iucunda uoluptas, réponse qu'il signale par son meminisse, me semble confirmer que le De rerum natura est bien à l'origine du motif gallien de la nuit d'amour comme moment de la naissance des liens entre amants. Properce décrit ces moments avec des motifs et termes galliens, indiqués probablement par le uidisse, et (ce qui constitue sa variation sur la fin du livre 4 du De rerum natura) en se mettant dans la même position de spectateur que Lucrèce, sauf qu'il n'est pas du tout un spectateur détaché : la iucunda uoluptas, c'est lui qui l'éprouve et il l'avait appelée de tous ses vœux ${ }^{57}$.

On retrouve le même traitement de la nuit d'amour dans l'élégie 1, 13, également adressée à Gallus, avec de nouveau le verbe uidi, qui est, sans doute, à prendre ici aussi comme un renvoi aux Amores:

Vidi ego te toto uinctum languescere collo et flere inectis, Galle, diu manibus (...)

Non ego complexus potui diducere uestros tantus erat demens inter utrosque furor. $(15-6 ; 19-20)$

«Moi je t'ai vu le cou complètement enlacé languir et pleurer longtemps les mains jetées sur elle (...) Moi je n'ai pu séparer vos étreintes, si grand était entre vous deux le délire de la fureur. "

31 Les motifs sont quasiment les mêmes que dans l'élégie 1, 10 : l'enlacement des corps, avec, comme c'était le cas pour la Vénus lucrétienne, une position dominatrice de la puella, et la sensation de mourir ou un alanguissement et des pleurs ... apparemment voluptueux. Le contraste avec Lucrèce est saisissant: au livre 4 le furor amoureux, associé au mot rabies, se marque par des pressions et agressions interprétées comme un désir secret d'arracher quelque chose aux membres de l'autre et/ou de faire intrusion à l'intérieur de son propre corps (1108-11). 
Le troisième texte où Properce a repris le motif gallien de la nuit d'amour, en s'opposant là encore à Lucrèce, est l'élégie, 2, 15, qui présente la particularité d'une prise de position aussi contre Virgile et sa condamnation de la passion élégiaque. L'analyse de ce texte va inaugurer la partie suivante de mon analyse, cette élégie comportant le développement d'un autre volet de l'utilisation par Gallus de la scène homérico-empédocléonne évoquée par Lucrèce : l'opposition amour/guerre.

\section{Empédocle à l'origine du motif élégiaque : 'faire l'amour, pas la guerre'?}

L'élégie 2, 15 s'ouvre sur la description d'une nuit d'amour, qui conjugue aux 'paroles' un combat (rixa, 4), un motif qui s'ajoute ici à celui des liens de l'amour, que l'on retrouve au vers 9: quam uario amplexu mutamus bracchia (« en combien d'embrassements variés nous avons changé la position de nos bras ») et aux vers 24-26, où l'on peut reconnaître des mots employés par Sulpicia (dies, uincire, catena et soluere), ce qui laisse supposer une source commune, autrement dit probablement un renvoi à Gallus :

Nox tibi longa uenit, nec reditura dies.

Atque utinam haerentis sic nos uincire catena uelles, ut numquam solueret ulla dies ! (24-6)

« Une longue nuit vient pour toi, et le jour ne reviendra pas. Et puisses-tu vouloir nous lier attachés par une chaîne telle qu'aucun jour jamais ne nous/la déliera. »

Ces renvois servent de préalable à un soutien de la poétique gallienne à la fois contre Virgile et contre Lucrèce. Les vers 29-30: Errat qui finem uesani quaerit amoris/ uerus amor nullum nouit habere modum («il se trompe celui qui cherche la fin d'un amour fou : le vrai amour ne sait pas avoir de mesure ») sont, en effet, un habile patchwork. Le verbe errat, Amor avec un qualificatif uerus, en position de sujet d'un verbe, renvoient à Gallus. Deux mots sont repris de Lucrèce : nouit (passim dans le De rerum natura) et finis (nimirum quia non cognouit quae sit habendi/ finis et omnino quoad crescat uera uoluptas; "assurément parce qu'il (le genre) humain ne connaît pas ce qu'est la fin de la possession et jusqu'où seulement s'accroît la vraie volupté ", 5, 1432-3). Modus fait écho à la Bucolique 10, où Ecquis erit modus ? ("Y aura-t-il une mesure (à ton amour) ", 28) est une question posée et implicitement un conseil donné à Gallus. Les vers 29-30 de l'élégie 2, 15 constituent une vive critique du point de vue commun à Lucrèce et à Virgile, pour qui la passion est condamnable précisément parce qu'elle ne connaît pas de limites. L'attaque contre l'épicurisme se poursuit avec les vers 39-40: Si dabit et multas, fiam immortalis in illis:/ nocte una quiuis uel deus esse potest (« si elle (Cynthie) m'en donnait plusieurs (il s'agit des nuits d'amour), je deviendrai au cours d'elles immortel: en une seule nuit n'importe qui même peut être un dieu »). Ce n'est pas exactement de cette façon-là qu'un épicurien accède un instant à l'état de bonheur 'sans mélange' qui est censé caractériser les dieux!

Ce qui différence le texte de Properce de ceux que j'ai cités jusque-là est que le motif de la nuit d'amour, sous l'aspect des liens sans limitation de durée produit par le uerus Amor, y est présenté, dans une espèce d'extension de la scène homérico-empédocléolucrétienne, comme la solution susceptible de mettre - pour toujours fin - aux guerres :

Qualem si cuncti cuperent decurrere uitam

et pressi multo membra iacere mero, 
non ferrum crudele neque esset bellica nauis, nec nostra Actiacum uerteret ossa mare, nec totiens propriis circum oppugnata triumphis lassa foret crines soluere Roma suos. (41-6)

"Si c'était cette vie-là que tout le monde désirait passer d'un bout à l'autre, et rester allongés les membres appesantis par beaucoup de vin, il n'y aurait pas de fer cruel, ni de navire de guerre, la mer d'Actium ne roulerait pas nos os, et Rome, attaquée de partout, ne serait pas lasse de dénouer ses cheveux tant de fois à cause de ses propres triomphes ». élégiaque, Properce la célèbre ici comme le moyen (trop beau pour être vrai ?) qui permettrait l'établissement définitif d'un 'bon' ordre du monde, caractérisé par le triomphe - définitif - de l'amour sur la Discorde ! Il est tentant d'y voir une réponse à la description virgilienne du bouclier d'Énée au livre 8 de l'Énéide, où une paix, présentée aussi comme définitive, est assurée par les armes grâce à la bataille d'Actium ${ }^{58}$ (671-713).

Le dernier texte propertien évoquant la nuit d'amour est l'élégie 3, 20. Il va me permettre de compléter la reconstitution des motifs galliens que j'ai tenté d'opérer. Properce y associe en effet un des grands motifs élégiaques : l'établissement de liens pérennes sous la forme de foedera amoris à la première nuit d'amour, caractérisée par l'alternance entre paroles et doux 'combats de Vénus' :

Nox mihi prima uenit ! primae da tempora nocti ! longius in primo, Luna, morare toro ${ }^{59}$.

Quam multae ante meis cedent sermonibus horae dulcia quam nobis concitet arma Venus !

Foedera sunt ponenda prius (...)

Namque ubi non certo uincitur foedere lectus, non habet ultores nox uigilanda deos,

et quibus imposuit, soluit mox uincla libido. (13-7; 21-3)

«La première nuit est venue pour moi ! Donne du temps à cette première nuit ; attarde-toi plus longtemps, lune, sur notre première couche. Combien d'heures céderont le pas à mes paroles avant que Vénus ne déclenche pour nous ses doux combats. Il faut d'abord établir un pacte (...) En effet quand une couche n'a pas de liens établis par un pacte sûr, une nuit à passer éveillés n'a pas de dieux pour la venger et pour ceux à qui il les a imposés, le désir les dénoue bientôt. »

Il est tentant de voir dans ces foedera un renvoi, via Lucrèce, à la métaphore empédocléenne de l'ôpkoc, échangé entre l'Amour et la Discorde (frg. B. 30, 3), un serment, qui, si on suit M. Garani ${ }^{60}$, était « thought of as something that literally binds and constrains ». Le mot foedus présentait en outre l'avantage d'avoir une connotation militaire : les foedera visent à mettre fin à la guerre, ce qui était le but visé par Vénus dans la scène lucrétienne ${ }^{61}$.

Quelques mots pour conclure sur l'opposition amour/guerre dans l'élégie gallienne. D'après ce que l'on peut reconstituer des Amores, elle s'y déclinait en deux cas de figure. D'un côté Gallus revendiquait le choix de l'amour à la place de la guerre. C'est la situation évoquée dans le papyrus de Qașr Ibrîm, où aux durs fata de l'amant, Gallus en l'occurrence, est opposée la gloire militaire de César :

Fata mihi, Caesar, tum erunt mea dulcia, quom tu maxima Romanae pars eri(s) historiae

postque tuum reditum multorum templa deorum fixa legam spolieis deiuitiora tueis. (fr. 2.2-5 Courtney) 
«Les destins alors, César, me seront doux quand tu seras la part la plus grande de l'histoire romaine et que je lirai qu'après ton retour les temples de nombreux dieux se sont enrichis parce que tes trophées y auront été accrochés ${ }^{62}$. » Il s'agit d'un texte liminaire donc programmatique. En tant que premier poème élégiaque du recueil, il reprend probablement certains motifs des Amores, avec des variations indiquant la modulation du genre propre à l'auteur. Quatre vers décrivent les situations opposées de Messala et du poète : le premier est en train de combattre, le second pris dans les liens amoureux :

Te bellare decet terra, Messala, marique

ut domus hostiles praeferat exuuias :

me retinent uinctum formosae uincla puellae

et sedeo duras ianitor ante fores. (53-6)

«Il te convient, Messala, de guerroyer sur terre et sur mer pour que ta maison étale

le butin pris sur l'ennemi ; moi, ce sont les liens de ma belle amie qui me retiennent

enchaîné, et je reste assis pour garder sa dure porte. ${ }^{63}$ "

L'allusion au butin est un équivalent de la mention des trophées accrochés dans les temples dans le papyrus de Qașr Ibrîm. Aux mots uinctum et uincla, utilisés aussi par Sulpicia et Properce, ce qui oriente vers une origine gallienne, s'ajoute ici retinent, qui est une variation sur un mot que Virgile met dans la bouche de Gallus dans la Bucolique 10 : detinet $(45)^{64}$.

Le second cas de figure présent dans les Amores à propos des rapports entre amour et guerre devait être la situation décrite dans le passage de la Bucolique 10, où Virgile fait parler Gallus, ce qui laisse supposer la reprise d'un motif célèbre de ce dernier. Gallus et Lycoris sont séparés parce qu'il a préféré la guerre à l'amour, une préférence condamnée par l'association du mot amor à Martis, et le choix de l'adjectif insanus pour qualifier cet amour ${ }^{65}$. De son côté Lycoris a aussi quitté Rome : elle est allée rejoindre dans les Alpes un autre amant. C'est une situation qui a été reprise également par Tibulle. Dans son élégie 1, 3, le poète est séparé de Délie. Parti à la guerre avec Messala, il est finalement retenu (tenet, 3) par la maladie en Phéacie. À l'inverse de Lycoris, Délie est restée à Rome et il l'espère chaste. On peut ajouter au dossier l'élégie 4,3 de Properce, où l'amant fait la guerre au loin, tandis que son amie l'attend à Rome. Le renvoi à Gallus y est appuyé par les noms choisis: Aréthuse et Lycotas (un nom construit sur la même racine que Lycoris), qui renvoient, l'un et l'autre, aux Amores. Une des innovations dues aux successeurs du fondateur du genre élégiaque sera de proposer avec le motif de la militia amoris un réinvestissement du genre de vie du miles dans celui de l'amant ${ }^{66}$. Cette thématique amorcée par Properce est poursuivie et amplifiée par Ovide ${ }^{67}$.

\section{Conclusion}

Il n'y a pas eu, me semble-t-il, dans les exemples de textes élégiaques que j'ai étudiés ici d'utilisation directe d'Empédocle. Il s'agit d'une réception au troisième degré, les deux premières étant constituées par la lecture empédocléenne de la scène homérique des amours de Mars et de Vénus et par la réception de cette scène dans le prooemium du De rerum natura. Pour Gallus le texte de Lucrèce a été un texte générateur, le philosophe ayant adjoint, par le biais des étymologies romaines du nom de Vénus, à l'allégorie 
physique une allégorie morale, et réutilisé cette grille de lecture dans ses réflexions sur l'amour au livre 4.

Paradoxalement le De rerum natura - ce sont les surprises de la réception - a joué un rôle déterminant dans l'invention par Gallus d'un nouveau genre littéraire, où il décrivait et justifiait un mode de vie dominé par le furor... ce qui était exactement ce que Lucrèce avait dénoncé et condamné. Il est résulté de cette transformation radicale des analyses lucrétiennes une réception d'Empédocle nouvelle. Les motifs de la 'victoire' de l'amour, des 'étreintes' et 'liens' érotiques, ainsi que l'antagonisme entre l'amour et la guerre ont été exploités par Gallus avec un instinct pour le choix des mots et un sens de la formule (à titre d'exemple : le célèbre uicit/uincit Amor) qui ne furent pas pour rien dans le succès du genre chez les poètes de la génération suivante. Cela explique aussi que, des Bucoliques à l'Énéide, Virgile ait jugé nécessaire de prendre position contre son protecteur et ami en défendant une autre lecture et de Lucrèce et d'Empédocle. C'est à cette lecture, qui prend dans son épopée une orientation nettement politique ${ }^{68}$, que les élégiaques ont répondu en se mettant à valoriser plus nettement que ne l'avait fait Gallus une vie consacrée à l'amour plutôt qu'à faire la guerre pour assurer la victoire et la 'paix' romaines ou encore - ce sera le cas d'Ovide dans les Métamorphoses - en finissant par proposer un autre type de réception d'Empédocle ${ }^{69}$.

\section{BIBLIOGRAPHIE}

Acosta-Hughes B., Stephen S. (2002) « Rereading Callimachus' Aetia fragment 1», CPh 97, 238-55.

Acosta-Hughes B., Stephen S (2013) Callimachus in context: from Plato to the Augustan Poets, Cambridge.

Bignone E. (1929) « Ennio ed Empedocle », RFIC 57, 10-30.

Bollack J. (1959) « Lukrez und Empedocles », Die neue Rundschau 70, 656-86.

Cairns F. (2006) Sextus Propertius. The Augustan Elegist, Cambridge.

Cameron A. (1995) Callimachus and his Critics, Princeton.

Campbell G.L. (2003) Lucretius on Creation and Evolution: a commentary on Lucretius, De Rerum Natura, 5, 772-1104, Oxford.

Clay D. (2000) « Recovering Originals : Peri Physeos and De Rerum Natura », Apeiron 33, 259-71.

Conte G. (1974) Memoria dei poeti e sistema letterario, Torino.

Conte G. (1984) « Aristeo, Orfeo e le Georgiche. Struttura narrativa e funzione didascalica di un mito », in Virgilio, il genere e i suoi confini », Milano, 43-53.

Conte G (2002) « Aristeo, Orfeo e le Georgiche : una seconda volta », in L'epica del sentimento, Torino, 65-89.

Courtney E. (1993) The Fragmentary Latin Poets, Oxford. 
Delvigo M.-L. (2011) Servio e la poesie della scienza, Pisa-Roma.

Dix T.K. (1995) «Vergil in the Grynean Grove : two riddles in the third Eclogue », Classical Philology 50, 256-62.

Fabre-Serris J. (2005) « Histoires d'inceste et de furor dans les Métamorphoses 9 et dans le chant en catalogue d'Orphée : une réponse d'Ovide au livre 4 des Géorgiques », Dictynna 2.

Fabre-Serris J. (2008) Rome, l'Arcadie et la mer des Argonautes. Essai sur la naissance d'une mythologie des origines en Occident, Lille.

Fabre-Serris J (2008b) « Ovide lecteur de Parthénios de Nicée », in Littérature et érotisme dans les Passions d'amour de Parthénios de Nicée, A. Zucker (ed), Grenoble, 189-205.

Fabre-Serris J (2009a) «D’Homère à Gallus : Protée, une variation virgilienne sur une figure poétique des Amores, Silène? ", in Protée en trompe-l'œil. Genèse et survivances d'un mythe d'Homère à Bouchardon, A. Rolet (ed), Rennes, 189-201.

Fabre-Serris J. (2009b) « Sulpicia: An/ other Female Voice in Ovid's Heroides A New Reading of Heroides 4 and 15 », Helios, 149-73.

Fabre-Serris J. (2013a) « Le Culex et la construction du mythe augustéen. Pratiques et enjeux d'un poème faussement adressé à Octave », in La costruzione del mito augusteo, M. Labate et G. Rosati (eds), Heidelberg, 285-302.

Fabre-Serris J (2013b) « Onomastics, Intertextuality and Gender. "Phyllis" in Roman Poetry (Gallus, Vergil, Horace, Propertius and Ovid) », in Roman Literature, Gender and Reception, D. Lateiner, B. Gold and J. Perkins (eds), London, 119-35.

Fabre-Serris J. (2013) « Genre et Gender : usages et enjeux de l'emploi de durus chez les élégiaques », Eugesta 3, 209-39.

Fabre-Serris (à paraître a), «The ars rhetorica : a Ovidian remedium for the female furor ? ", in Augustan Poets and the Irrational, P. Hardie (ed), Oxford University Press, Oxford.

Fabre-Serris (à paraître b) « Enjeux moraux et idéologiques des usages d'Empédocle au livre 15 des Métamorphoses : une réponse d'Ovide à Virgile (Enéide 6 et 8) », in Les présocratiques dans la poésie latine, C. Lévy et S. Franchet d'Esperey (eds), Paris.

Farrell J. (1991) Vergil's Georgics and the traditions of ancient epic : the art of allusion in literary history, Oxford.

Friedländer, R. (1993c) « The pattern of sounds and atomistic theory in Lucretius », AJP 62, 13-34.

Furley D. (1970) « Variations on Themes from Empedocles in Lucretian's Proem », B.I.C.S. 17, 55-64.

Gale M. (1994) Myth and poetry in Lucretius, Cambridge.

Gale M. (1994 b) «Lucretius 4, 1-25 and the Proems of the De Rerum Natura », PCPhS 40, 1-17.

Garani M. (2007) Empedocles Redivivus : Poetry and Analogy in Lucretius, New-York-London.

Garani M. (2007) « Cosmological Oaths in Empedocles and Lucretius », in Horkos, the Oath in Greek Society, A.H. Sommerstein and J. Fletcher (eds), Exeter, 189-202.

Garani M. (2011) « Revisiting Tarpeia's myth in Propertius (IV, 4) », Leeds International Classical Studies, 10.3.

Garani M (à paraître) « The advent of Maiestas (Ovid, Fasti, 5.11-52) », in From Middles to New Beginnings, A. Michalopoulos and S. Papaioannou (eds), Newcastle, 143-78. 
Gill C. (1983) « Did Chrysippus understand Medea ?», Phronesis 28, 136-49.

Hardie P. (1986) Virgil's Aeneid. Cosmos and Imperium, Oxford.

Hardie, P. (1995) « The Speech of Pythagoras in Ovid Metamorphoses 15 : Empedoclean Epos », CQ, 201-14.

Hardie P. (2009) Lucretian Receptions: History, The Sublime, Knowledge, Cambridge.

Hinds S. (1984) « Carmina digna. Gallus P. Qașr Ibrîm 6-7 metamorphosed », Liverpool Latin Seminar IV, 43-54.

Hinds S. (1987) « The Poetess and the Reader : Further Steps towards Sulpicia », Hermathena 143, $29-46$.

Hinds S. (1998) Allusion and intertext: Dynamics of Appropriation in Roman Poetry, Cambridge.

Hinds S. (2006) « Venus, Varro and the vates: toward the limits of etymologizing interpretation», Dictynna 3.

Hunter R. (2006) The Shadow of Callimachus. Studies in the reception of Hellenistic poetry at Rome, Cambridge.

Hutchinson G (2001) « The Date of the De Rerum Natura», CQ 51, 150-62.

Inwood B. (2001) The Poem of Empedocles, University of Toronto Press, Toronto.

Keith A. (2008) Propertius, Poet of Love and Leisure, London.

Kennedy D. (1982) « Gallus and the Culex », C.Q. 32, 371-89.

Klein F. (2005) «Rursus pomi iactu remorata secundi (Mét.X, 671). La mora et la poétique ovidienne de la brièveté », Dictynna 2.

Klein F. (2012) « Tradition épique et modèles hellénistiques dans le livre XIV des Métamorphoses », R. É.L. 90, 195-212.

Klein F. (2014) « Les catégories stylistiques des poètes augustéens, entre théorie esthétique et caractérisation générique? ", in L'Héröque et le Champêtre. Vol. I. Les catégories stylistiques dans le discours critique sur les arts, M. Cojannot-Leblanc, C. Pouzadoux et É. Prioux (eds), Presses Universitaires de Paris Ouest, 125-50.

Knox P. (1986) Ovid's Metamorphoses and the Augustan Poetry, Cambridge.

Maltby R. (1993) «The limits of etymologizing », Aevum Antiquum 6, 257-75.

Martin A. and Primavesi O. (1998) L'Empédocle de Strasbourg (P. Strasb. Gr. Inv. 1665-1666), Strasbourg/Berlin.

Martín Rodríguez A.M. (2002) De Aedón a Filomela. Génesis, sentido y comentario de la versión ovidiana del mito, Las Palmas de Gran Canaria.

Miller J. (1993) « Ovidian Allusion and the Vocabulary of Memory », M.D. 30, 153-64.

Nelis D. (2001) Vergil's Aeneid and the Argonautica of Apollonios Rhodius, Leeds.

Nelis D. (2004) « Georgics 2. 458-542. Virgil, Aratus and Empedocles», Dictynna 1.

Nelis D. (2009) « Ovid's Metamorphoses 1. 416-51 : noua monstra and the foedera naturae », in Paradox and the Marvellous in Augustan Literature and Culture, P. Hardie (ed), Oxford, 248-67.

Norden E. (1915) Ennius und Vergil, Leipzig. 
Parker H.N. (1994) « Sulpicia, The Auctor de Sulpicia and the Authorship of 3, 9 and 3, 11 of the Corpus Tibullianum », Helios, 9-62.

Ross D. (1975) Backgrounds to Augustan Poetry. Gallus, elegy and Rome, Cambridge.

Rothaus Caston R. (2006) « Love as Illness: poets and philosophers on romantic love », C.J. 103, 1, 271-98.

Sedley D. (1989) « The Proems of Empedocles and Lucretius », GRBS 30, 269-96.

Sedley D. (1998) Lucretius and the Transformation of Greek Wisdom, Cambridge.

Sedley D. (2003) « Lucretius and the New Empedocles », Leeds International Classical Studies, 2, 4.

Sedley D. (2007) «The Empedoclean Opening », in Lucretius, M. Gale (ed), 48-87.

Snyder J. (1980) Poetry and Reception in Lucretius' De rerum natura, Amsterdam.

Tränkle H. (1963) « Elegisches in Ovid's Metamorphosen », Hermes 91, 465-76.

Trépanier S. (2004) Empedocles. An interpretation, Routledge, New York \& London.

Wright M.R. (1981) Empedocles: The Extant Remains, New Haven.

\section{NOTES}

1. Voir en particulier Hardie (1986), Sedley (1989, 2003, 2007), Farrell (1991), Nelis (2001, 2004, 2009). M. Garani (2011 et à paraître) a travaillé sur Properce et Ovide.

2. Callimaque, Aitia, fr. 1, 1-4, Pfeiffer.

3. Callimaque, Aitia, fr. 1, 21-4, Pfeiffer.

4. Voir la réception de Callimaque, voir Cameron (1995), Acosta-Hughes and Stephen (2002 et 2013), Klein (2012 et 2014).

5. Je suis moins prudente que Hunter $(2006,21-3)$ sur le renvoi à Gallus dans ce texte et dans celui de Properce.

6. On trouve dans les vers 1-8 de l'élégie 2,13 de Properce des détails similaires : une allusion à Hésiode incluant l'adjectif Ascraeus (Ascraeum ... nemus, 4), l'évocation du pouvoir traditionnellement attribué à Orphée à travers la mention des arbres qui suivent le poète et des bêtes sauvages qu'il attire (non ut Pieriae quercus mea uerba sequantur/ aut possim Ismaria ducere ualle feras), ainsi que le nom de Linus (8), le tout dans un contexte où est affirmée, comme dans le papyrus de Qașr Ibrîm, l'importance de la domina en tant que destinataire des vers (sed magis ut nostro stupefiat Cynthia uersu,7). En raison de cette thématique générale inspirée des Amores, on peut supposer que les éléments communs entre Properce et Virgile (vu le contexte gallien de leur utilisation dans la Bucolique 6) renvoient eux aussi à un ou à des passages des Amores.

7. On trouve les noms d'Orphée et de Linus, avec un motif présent aussi chez Properce (la supériorité d'un poète sur un autre), à la fin de la Bucolique 4 dans un contexte gallien constitué, entre autres, par un autre écho du papyrus de Qașr Ibrîm (Arcadia ... iudice/ se iudice, 58-9).

8. Pour une liste plausible de termes tirés des Amores et utilisés par les poètes latins pour renvoyer à Gallus, voir Cairns (2006) et Fabre-Serris (2008). Il est par ailleurs probable (c'est ce qui ressort de la confrontation de certains textes de Virgile, Properce et Ovide) que l'interprétation générique du prologue des Aitia, que l'on trouve au début de l'églogue et qui sera une constante dans la poésie latine, remonte à Gallus, qui aurait opposé à la poésie épique le genre qu'il venait d' 'inventer' : l'élégie érotique (voir Fabre-Serris, 2008a, 58, n. 18).

9. Voir Cairns (2006, 123, 126-7, 134 n. 100, 171 n. 72) et Fabre-Serris (2008a, 93-4, 98, 127, 175). 
10. Il y a là un jeu avec Gallus, que Virgile renouvellera au livre 4 des Géorgiques, Orphée étant probablement un des personnages associés dans les Amores à des mises en scène de la valeur et des particularités des choix poétiques de leur auteur.

11. Tum uero in numerum Faunosque ferasque uideres/ ludere, tum rigidas motare cacumina quercus (" alors on aurait pu voir les Faunes et les bêtes sauvages s'ébattre en cadence, alors les chênes raides faire mouvoir leurs cimes »).

12. Il est question ensuite du parcours - fixe - des astres, de la lune et du soleil dans le ciel (un des objets de la poésie scientifique). Puis la perspective change et devient mythologique avec la mention des nymphes accolée à celle des fleuves dans une liste qui comprend aussi les monts et les animaux. Cette dimension mythologique se poursuit avec l'évocation des couples divins qui régnèrent successivement sur l'univers: Ophion et Eurynomé, puis Rhéa et Cronos durant l'enfance de Zeus.

13. Sur la connotation lucrétienne de semina et exordia, voir Delvigo (2011,60-1), auxquels on peut ajouter aussi anima, paulatim et concrescere (utilisé 18 fois chez Lucrèce). Sur le fait que Lucrèce reprochait aux philosophes comme Empédocle, Héraclite et Anaxagore, l'absence du vide dans leurs théories sur l'origine du monde voir, aussi Delvigo (2011, 72).

14. Voir par exemple à la suite de Furley, Sedley $(2007,65)$ : "The very idea of using individual phenomena like sea, rain, wind, and sun to symbolize the four elemental stuffs is thoroughly Empedoclean ».

15. Pyrrha, Saturne, Prométhée étaient présents chez Hésiode, si l'on en juge, pour les deux derniers, par la Théogonie et pour l'histoire de Deucalion et Pyrrha par un fragment (Catalogue frg. 3 Rzach). Le schéma du chant est par ailleurs hésiodique au sens large : on a la même succession de motifs cosmogoniques et érotiques dans la Théogonie, à qui les Anciens associaient le Catalogue des femmes.

16. Voir la note 6.

17. Voir Ross (1975, 72-3).

18. Lucrèce, De rer. nat., 3, 420. Sur l'usage de l'expression chez Gallus et les poètes augustéens, voir Hinds (1984).

19. Le personnage de Médée a particulièrement intéressé les Stoïciens pour son furor. Voir Gill (1983).

20. C'est ce que l'on peut supposer quand on confronte les catalogues d'erotika pathemata de Properce dans l'élégie 3, 19 et d'Ovide dans l'Ars Amatoria, 1, 285-340, deux textes sous influence gallienne dans la mesure où il s'agit de modèles réduits du recueil de Parthenius de Nicée, dédié à Gallus. L'histoire de Médée fait partie des mythes communs aux deux textes (Properce, 3, 19, 17-8; Ovide, Ars Am., 1, 336). Le troisième texte énumérant des erotika pathemata qu'on peut ajouter à cette liste est précisément la Bucolique 6, qui a d'autres histoires en commun avec les textes de Properce et d'Ovide. Voir Fabre-Serris (à paraître a).

21. En supposant que les jeux intertextuels dont est tissée la Bucolique 6 sont basés sur des renvois à la réception d'Empédocle chez Apollonios de Rhodes, chez Lucrèce et chez Gallus, je me situe dans une ligne interprétative proche de celle de Garani, qui remarque (2007, p. 17) : « More generally, a detailed comparison between Empedocles and Lucretius should encourage the reading anew of Latin texts which condense simultaneous allusions to Empedocles and Lucretius, as well as echoes from other texts which are much indebted to Empedocles, such as Aratus, Apollonios Rhodius, the allegorists and Ennius. In this way more light will be shed upon Empedocles' reception by the Roman epicists, especially Virgil and Ovid, and it will become clearer why Lucretius was read as Empedocles Romanus par excellence in the Roman tradition of scientific didactic epos what Hardie sees as 'empedoclean epos' ", quoique sa perspective ne soit pas la même que la mienne puisque ce qui l'intéresse au premier chef, ce sont les rapports entre Empédocle et Lucrèce. 
22. Le Permesse est aussi mentionné dans l'élégie 2, 10 de Properce, qui l'oppose en tant que lieu symbolique d'une inspiration érotique (élégiaque) aux sources d'Ascra, considérées traditionnellement comme le lieu de l'inspiration hésiodique (épique) : Nondum etiam Ascraeos norunt mea carmina fontes,/ sed modo Permessi flumine lauit Amor (« mes chants ne connaissent pas encore les sources d'Ascra ; l'Amour les a seulement baignés dans le fleuve du Permesse », 25-6). Voir Cairns (2006, 125-6; 329-30), qui commente ainsi ce texte: "In Propertius' case the Permessus is explicitly associated with his own erotic elegy, inspired by Gallus, since it is Amor who 'has bathed' Propertius in the Permessus. Gallus may have designated his poetry written prior to his Heliconian imitation as erotic with similar explicitness » (330).

23. Fabre-Serris (2005 et 2013a).

24. Fabre-Serris (2013b).

25. Fabre-Serris (2005).

26. Voir par exemple Kennedy (1982).

27. On peut postuler une origine gallienne commune à ces deux descriptions de bois dans la mesure où dans leurs contextes (le chant d'Orphée et l'ensemble du Culex), Ovide et l'auteur du Culex renvoient à Gallus mais pas aux mêmes motifs ou termes considérés par la critique comme galliens, ce qui permet d'écarter l'hypothèse de renvois directs de l'auteur du Culex au seul texte d'Ovide.

28. Selon Cairns (2006, 232 ; 235-49), il est possible que l'histoire d'Hylas ait été traitée aussi par Gallus.

29. La version choisie par Virgile diffère de celle qu'adoptera Ovide. Voir Martín Rodríguez (2002).

30. Sur la probabilité pour que Pasiphaé ait été une des héroïnes mythologiques évoquées dans les Amores, voir Fabre-Serris (en cours de publication a).

31. Le fait que l'ensemble du chant de Silène soit, dans les derniers vers, présenté comme une œuvre composée par Apollon quand il était sur les bords de l'Eurotas, autrement dit, quand il était amoureux d'Hyacinthe, est une ultime allusion à Gallus. Si on se réfère à l'élégie 2, 34, 91-2 de Properce, Gallus aurait évoqué ce mythe traité par Euphorion (voir Knox, 1886, 15-6, qui se réfère à Tränkle, 1963, 22-3). Si l'on accepte l'interprétation de la Bucolique 3 proposée par Dix (1995), le cadre en était son poème sur le bois de Grynium.

32. Sedley $(2007,72)$.

33. Sur les allusions à Gallus, voir Conte (1984 et 2002).

34. Le mot cura désigne les soins, le souci, mais aussi leur objet : autrement dit, la femme aimée. C'est sans doute, dans ce dernier sens, un mot du glossaire gallien si l'on en juge par l'expression tua cura Lycoris (Buc. 10,22) et diverses utilisations du mot chez Properce. Voir à ce sujet Cairns

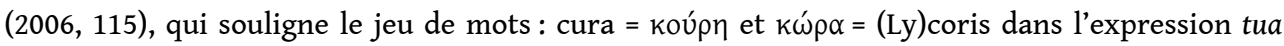
cura Lycoris.

35. Les amours de Mars et de Vénus sont sans doute aussi mis en position de modèle pour tous les autres amours des dieux.

36. Sur les autres traits rapprochant Protée et Silène, voir Fabre-Serris (2009a).

37. Voir la préface des Erotika pathemata.

38. Fabre-Serris (2008b, 194-200). Je ne peux pas entrer ici dans le détail, mais les termes utilisés par Properce ont aussi une connotation gallienne (voir Fabre-Serris (2008a, 94).

39. Comme me l'a fait remarquer G. Rosati, on trouve le même procédé dans l'élégie 2, 30. Jouant sur le uincit/uicit Amor gallien, Properce y argue d'autres exempla d'amours chez les dieux (l'amour de Jupiter pour Sémélé et pour Ganymède), afin d'appuyer l'idée - centrale dans l'élégie - que rien ni personne ne peut vaincre l'Amour et que lui-même donc ne peut être accusé d'y céder : quod si nemo exstat qui uicerit Alitis arma,/ communis culpae reus unus agor? (" s'il ne s'est jamais trouvé personne pour triompher des armes du dieu ailé, serai-je le seul à être accusé d'une faute commune à tous ?", 31-2). 
40. Furley (1970) considère que ces échos sont la marque de la reconnaissance d'une dette philosophique. La thèse développée par Sedley (2007 par exemple) est que le prooemium de Lucrèce est calqué sur celui d'Empédocle en qui il salue ainsi un prédécesseur sur le plan uniquement littéraire et poétique.

41. "Lucretius is imitating Empedocles' proem, but adapting it, as he goes along, (a) to a Roman patriotic theme, and (b) to Epicurean philosophy, at the same time steering us gently away from Empedocles' actual doctrines. His object? To announce himself as the Roman Empedocles - the great Roman poet of nature. In short his is laying claim to a literary, not a philosophical heritage »(Sedley, 2007, 74).

42. Pour le point de vue inverse, voir par exemple Bollack (1959), Furley (1970), Gale (1994 b), Clay (2000), Campbell (2003), Garani (2007b).

43. C'est ce jeu que d'ailleurs Sedley s'attache à éclaircir quand il se livre à un relevé des différents renvois à Empédocle pour fonder son hypothèse d'un hymne à Aphrodite. Cela l'amène à supposer que la vision que Lucrèce donne de Vénus en train de prier Mars d'intervenir en faveur des Romains pourrait être inspirée par une scène où "Aphrodite as Love is asked to propitiate Ares as Strife » $(2007,79)$ et que l'adaptation lucrétienne consisterait en une reprise accompagnée d'un correctif épicurien : "that Venus' hoped-for propitiation of Mars represents no more than people's return to the one true conception of the divine nature as tranquill and detached instead of angry and warlike ». Comme il le reconnaît : "the connexion of thought could no doubt have been made clearer »; personnellement je ne suis pas vraiment convaincue qu'il faille comprendre que le message adressé aux Romains est « let your belief in a peaceful god overcome your belief in a warlike god because peacefulness is the true essence of godlike happiness ». On peut faire plutôt l'hypothèse, comme le propose Garani à propos d'échos empédocléens dans le vocabulaire utilisé par Lucrèce, qu'à certains endroits de son poème, Lucrèce reconnaissait «points of philosophical agreement between Empedocles and Epicurus that (could) be justified by their common anti-Parmenidean heritage » $(2007 \mathrm{~b}, 9)$.

44. C'est ce que souligne à juste titre Trépanier $(2004,40):$ «Indeed, how can Sedley reasonably exclude the possibility that Lucretius meant the hymn to Venus to be recognizably Empedoclean, but not necessarily an adaptation or partial translation of Empedocles' own proem ? Even within the tradition of aemulatio, there is a wide enough spectrum of originality available to the Roman poet, and the new work need not be either a translation, or a Greek scheme with Roman accretions. ».

45. Trépanier (2004, 40 et 207, n. 29).

46. Pour Gale $(1994,72)$ par exemple, la variation lucrétienne consiste en une correction d'Empédocle: l'union de Mars et de Venus «may be a way of suggesting that Empedocles' creative and destructive forces are in fact eternally and indissolubly linked and that his theory of alternation on a cosmic scale is superfluous ».

47. Hinds, (2006, Par. 47). Pour récapituler : « To negotiate with the divine, to comprehend the divine, to control the divine, one must embrace the divine in its totality. In the case of Venus our "etymological catechism" operates to ask and answer a series of questions. Who is the binder? Who is the conqueror? Who has the force? Who comes to all ? Who is the granter of indulgence ? Whom do we venerate? Who is the dispenser of drugs and charms? To all of these questions the response is "Venus' " (Par. 49).

48. Hinds (2006, Par. 25 et 26). Ce faisant il prend la suite, comme il le souligne, de Snyder (1980, $106-7$; 114-6, 133-4) pour qui l'étymologie de Vénus par uenire est ensuite doublée par son étymologie avec uenenum.

49. «t'étant avec ton corps sacré répandu tout autour par-dessus lui qui est allongé. »

50. L'insistance sur le motif des liens de Vénus me paraît de nature à conforter l'hypothèse de renvois de Lucrèce à Empédocle par le biais des étymologies varroniennes du nom de la déesse : la conception empédocléenne de l'Amour en tant que force d'union pouvant être rapprochée de 
l'idée, clairement théorisée par Varron, selon laquelle Vénus est une force qui lie : et mas ignis, quod ibi semen, aqua femina, quod fetus ab eius humore, et horum uinctionis uis Venus (" le feu est mâle, ce qu'est par ailleurs la semence, l'eau est femelle, parce que le fœtus se développe à partir de son humidité, et la force qui les unit c'est Vénus » De lingua latina 5, 61).

51. Ennius avait probablement donné une couleur empédocléenne au discours de 'son' Homère. Voir Garani $(2007$ b, 26-7), qui cite Hardie $(1986,78-9)$ : « the general sequence of natural philosophy followed by history may have been designed to provide a generalized cosmic overture for the detailed Roman themes that followed » et qui poursuit « From this point of view, Ennius should be considered the decisive intermediary for the introduction into later Roman literature of Empedocles' integration of cosmological-philosophical order with historical. »

52. Voir Norden (1915, 10-30), Bignone (1929, 22-5).

53. C'est une hypothèse développée par Garani : voir infra.

54. Sur les textes de l'amicus Sulpiciae, voir Hinds (1987), Parker (1994), Fabre-Serris (2009b).

55. Je pense, comme Parker (1994), que les élégies 3, 9 et 3, 11, écrites à la première personne sont de Sulpicia (Fabre-Serris 2009b).

56. Sur meminisse comme signal intertextuel, voir Conte (1974), Miller (1991), Hinds (1998).

57. Je ne développe pas sur la suite de la variation que constitue l'élégie 1, 10. Je souligne simplement que le renvoi à Lucrèce permet à Properce de tenir sur l'amour un discours de praeceptor, qui est la position qu'il adopte par rapport à Gallus dans la monobiblos où il valorise sa propre expérience élégiaque comme plus totalisante que celle de son modèle et ami. Le vers 17 : et possum alterius curas sanare recentis (« et je peux soigner les blessures récentes d'un autre »; 17) est aussi une allusion à Lucrèce : uolginagaque uagus Venere ante recentia cures, « (si) tu (ne) soignes (pas) auparavant, en allant à l'aventure, par des amours vagabondes, des blessures blessures" 4, 1071). Sur le fait que ce texte est à lire "as Propertius' meditation on the relationship of his amatory elegy to that of Gallus », voir Keith $(2008,121-2)$.

58. Virgile y signale le rôle de Mars (700) et de la Discorde (702).

59. Il y a des chances pour que le mot mora ait fait partie du glossaire gallien. Nous avons vu qu'on le trouvait dans l'élégie 1, 10 (cum te complexa morientem, Galle, puella/ uidimus et longa ducere uerba mora, 5-6). Sur la mora comme concept stylistique et poétique chez Ovide, voir Klein (2005).

60. Garani (2007b, 177 ; voir aussi 239, n. 99) explique ce sens par le fait que « the word ópкoৎ is

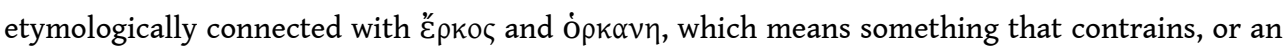
enclosure "; " it seems very likely that, following Epicurus' theory of language, Lucretius looks

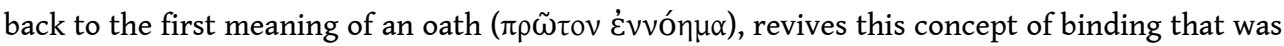
already latent in Empedocles' oath and transfers it to the foedera that, as it has already been argued above, he employs as the Latin equivalent to the Greek metaphor ». Ce passage reprend une discussion plus développée dans Garani (2007a).

61. Sur la possibilité qu'il y ait, dans le vers 40 petens placidam Romanis ... pacem, une allusion aux guerres civiles, voir Hutchinson (2001).

62. Sur le fait que «Gallus mettrait ici en regard de sa propre pratique de l'élégie, liée à sa préférence pour l'amour, malgré ses souffrances, la prose ou l'épopée historique, qui célèbrent les guerres, leurs généraux et le destin de Rome », voir Fabre-Serris $(2013,225)$.

63. Le dernier vers introduit une variation.

64. On peut évoquer comme variation sur cette situation l'élégie 1, 6 de Properce, qui s'adresse aussi à un homme de guerre, Tullus, et fait le même choix de vie que Tibulle, avec des mots renvoyant également au papyrus. On trouve au vers 26 nequitiae (Gallus, fr. 2,1 Courtney), au vers 30 fata (Gallus, fr. 2,2), et au vers 34 pars imperii, qui est une variante de pars historiae (Gallus, fr. 2, 3).

65. Si le passage évoquant Lycoris correspond à un propemptikon, Virgile a probablement ici mixé deux passages différents des Amores. 
66. Pour une analyse plus développée de ces deux motifs galliens et de leurs reprises et variations chez les élégiaques (en particulier sur la façon dont ils ont cherché à réinvestir le genre de vie du soldat dans celui de l'amant), je renvoie à mon étude sur les usages de l'adjectif durus, selon qu'il est appliqué au uir ou à la puella (Fabre-Serris, 2013).

67. Voir Murgatroyd (1975), Cahoon (1988), McKeown (1995), Gale (1997), Fabre-Serris (2013).

68. Voir les différentes études de P. Hardie et D. Nelis.

69. Voir par exemple Hardie (1995), Fabre-Serris (2013 et en cours de publication b).

\section{RÉSUMÉS}

Ce papier vise à montrer, à partir d'une série d'analyses de textes de Virgile, de Lucrèce et des poètes élégiaques, que la lecture faite par Gallus, non d'Empédocle directement, mais de la réception de ce dernier chez Lucrèce a joué un rôle fondamental dans la genèse de grands motifs élégiaques. À ceux du furor et de l'absence de remèdes à l'amour,on peut ajouter en effet le déploiement de deux des étymologies de Vénus exploitées par Lucrèce dans sa version de l'épisode homérique des amours de Mars et de Vénus perçu par les Anciens comme empédocléen : uincere et uincire. La première étymologie a donné lieu à la thématique de la victoire de l'amour et des combats de Vénus opposés aux guerres menées par les Romains, la seconde à celle de la première nuit d'amour, qui 'lie' les amants et initie le début de foedera souhaités pérennes mais soumis à d'incessantes fluctuations.

\section{INDEX}

Mots-clés : amicus Sulpiciae, amours de Mars et de Vénus, antagonisme amour/guerre., combats de Vénus, étymologies de Vénus, foedera, Gallus, Lucrèce, nuit d'amour, Ovide, Properce, Réception d'Empédocle, Sulpicia, Tibulle, uincere, uincire, Virgile

\section{AUTEUR}

\section{JACQUELINE FABRE-SERRIS}

Université Charles-de-Gaulle-Lille 3

Centre Halma-Ipel UMR 8164 